\title{
BMJ Open Use of antibiotics and colorectal cancer risk: a primary care nested case-control study in Belgium
}

\author{
Johannes Van der Meer (1D , ${ }^{1}$ Pavlos Mamouris, ${ }^{2}$ Vahid Nassiri, ${ }^{3}$ Bert Vaes (D) , \\ Marjan van den Akker (D) 4,5
}

To cite: Van der Meer J, Mamouris P, Nassiri V, et al. Use of antibiotics and colorectal cancer risk: a primary care nested case-control study in Belgium. BMJ Open 2021;11:e053511. doi:10.1136/ bmjopen-2021-053511

- Prepublication history for this paper is available online. To view these files, please visit the journal online (http://dx.doi. org/10.1136/bmjopen-2021053511).

Received 26 May 2021 Accepted 17 November 2021

Check for updates

C Author(s) (or their employer(s)) 2021. Re-use permitted under CC BY-NC. No commercial re-use. See rights and permissions. Published by BMJ.

For numbered affiliations see end of article.

\section{Correspondence to}

Professor Marjan van den Akker; m.vandenAkker@ allgemeinmedizin.uni-frankfurt. de

\section{ABSTRACT}

Objectives To examine the association between the use of oral antibiotics and subsequent colorectal cancer risk. Design Matched case-control study.

Setting General practice centres participating in the Integrated Computerised Network database in Flanders, Belgium.

Participants In total, 1705 cases of colorectal cancer diagnosed between 01 January 2010 and 31 December 2015 were matched to 6749 controls by age, sex, comorbidity and general practice centre.

Primary outcome measure The association between the number of prescriptions for oral antibiotics and the incidence of colorectal cancer over a period of 1-10 years estimated by a conditional logistic regression model.

Results A significantly increased risk of colorectal cancer (OR 1.25, 95\% Cl 1.10 to 1.44) was found in subjects with one or more prescriptions compared with those with none after correction for diabetes mellitus. No dose-response relationship was found.

Conclusions This study resulted in a modestly higher risk of having colorectal cancer diagnosed after antibiotic exposure. The main limitation was missing data on known risk factors, in particular smoking behaviour. This study did not allow us to examine the causality of the relationship, indicating the need of further investigation.

\section{INTRODUCTION}

The development of antibiotics during the 20th century has had a ground breaking impact on modern medicine. ${ }^{1}$ However, their administration is known to be associated with the development of short-term gastrointestinal symptoms such as diarrhoea. ${ }^{2}$ While these conditions are generally self-limiting, both oral and intravenous use of antibiotics have been linked to a more permanent state of distorted colonic balance, one example being a Clostridium difficile infection. ${ }^{3}$ The human gut contains a diverse microbial community and has a crucial role in the defence against pathogenic bacteria, a balance that can be influenced by numerous factors. ${ }^{4}$ Little is known about long-term effects of oral antibiotic exposure, for example, in the relationship between antibiotic exposure and

\section{Strengths and limitations of this study}

- This case-control study was sufficiently powered to detect an association between antibiotic prescriptions and colorectal cancer.

- The large data set minimised selection bias.

- This study provided us an insight into the prescription behaviour of the general practitioner.

- Smoking and obesity, known risk factors for colorectal cancer, were missing in Integrated Computerised Network database. These potential confounders could not be taken into account in our study.

development of colorectal cancer (CRC). A systematic review of the link between carcinogenesis and microbial dysbiosis suggests a relationship, yet hard conclusions about causality could not be stated. In particular, there are hints that the metabolic environment is involved, which can create a proinflammatory state. ${ }^{5}$ In addition a persistent altered microbiotic state after oral antibiotic exposure was shown by Dethlefsen and Relman 10 months after initial exposure. ${ }^{6}$ Human studies with a clear link between an altered colonic state and colorectal oncogenesis in vivo were not found.

The development of CRC in general is typically a multifactorial process with a development time of over 10 years and is dependent on modifiable and unmodifiable factors. ${ }^{78}$ Having a first-degree relative with CRC and having an inflammatory bowel disease (IBD) are major risk factors. ${ }^{8}$ Minor risk factors are smoking, ${ }^{9}$ abdominal obesity, ${ }^{10}$ lack of physical activity, diabetes mellitus (DM), male sex and increased age. ${ }^{8}$ Roughly $3 \%-5 \%$ of CRC is a hereditary form. ${ }^{8}$ Whether exposure to oral antibiotics might be one of these factors is a complex association to investigate.

The importance of investigating the potential presence of such a relationship can be demonstrated by the current disease burden caused by the development of CRC, 
which is significant by measurement of incidence and consequence. For instance in Europe it is estimated to account for $12 \%$ of all cancers and cancer-related deaths annually. ${ }^{11}$ Analysis of geographical distribution between 21 European countries during the first 2 decades of the 21st century resulted in great intercountry differences. ${ }^{12}$ Outpatient antibiotic use also differs greatly between countries in this region. This was shown by an analysis of 33 European countries resulting in a factor of 3.8 when comparing the highest and lowest national consumption. ${ }^{13}$ Considering the heterogeneous geographical distribution of both antibiotic use and CRC incidence an investigation in a country where this has not yet been analysed yet might be of additional value.

Our aim is to evaluate the association between oral antibiotics prescribed in Flemish general practice and subsequent diagnosis of CRC with correction for comorbidity including DM, an established risk factor for CRC. ${ }^{8}$

A thorough search with this focus can be summarised by describing the results of three meta-analyses, which combined analysed a total of 11 individual case-control and cohort studies. ${ }^{14-24}$ In 2019 Syanolu et a ${ }^{25}$ investigated a total of eight studies and their quantitative synthesis resulted in a significant OR of 1.20 (95\% CI 1.10 to 1.32) when cumulatively assessing the number of prescriptions. In their conclusion they consider a weak association between exposure and outcome but no clear signs for a dose-response relationship. The other two meta-analyses were both published in 2020, were both based on the same 10 studies and resulted in comparable results. Qu et $a l^{26}$ analysed a total of over 4.8 million participants, which resulted in an OR of 1.09 (95\% CI 1.02 to 1.17). In particular, the additional analysis of anti-aerobic antibiotics showed an increased risk. Lastly, Simin $e t a l^{27}$ compared ever users to none users which resulted in an OR of 1.17 (95\% CI of 1.05 to 1.30). Limitations mentioned by these meta-analyses are the high heterogeneity between studies in terms of measurement of exposure, measurement of outcome, differing antibiotic use per country and potential biases such as residual confounding. In addition to the already present studies, ours will add weight in its geographical location since this is the first study in Belgium, with its own prescription behaviour and CRC diagnostic procedures and incidence.

\section{METHODS}

\section{Study design and context}

For this nested case-control study we used the Belgium based Integrated Computerised Network (Intego). Since its foundation in 1994 general practitioners (GPs) have recorded over 3 million diagnoses and 12 million prescriptions. It covers more than $2 \%$ of the Flemish population and is representative for the Flemish population in terms of age and gender distribution. ${ }^{28}$

\section{Data}

Registered details contain information about the subjects' age, gender, general practice and date of prescriptions and diagnosis. The latter two were coded using the Anatomical Therapeutic Chemical (ATC) classification system and the International Classification of Primary Care (ICPC-2), respectively. ${ }^{28}$

\section{Case and control selection}

A flow diagram of the patient selection is presented in figure 1 . All patients aged above 18 years old registered in the Intego database during the period of 01 January 2010 to 31 December 2015 (hereinafter called the selection interval) were eligible for inclusion. Cases were those with a first diagnosis of CRC (ICPC-2 code D75) registered by their GP during the selection interval. The index date used for the CRC cases was equal to the date of their first diagnosis. Everyone with the presence of CRC prior to the selection interval was excluded. Furthermore, subjects with IBD prior to the index date were excluded, since IBD might distort the association between antibiotics and CRC. $^{8}$

Each case was matched to four optimally chosen control subjects matched on age $( \pm 5$ years $)$, sex, the number of comorbidities ( \pm 1 disease) and general practice. Controls were assigned the same index date as their case counterpart. Mamouris et al developed an optimal algorithm to match cases and controls in an optimal, fast and efficient way. This algorithm is efficient, since it accommodates replacement with or without controls; fast, since it is executable in seconds even with millions of controls; and optimal, since the closest control is always captured. Specifically, in the scenario that a case has only one control we assured that this control will be matched to this case, thus maximising the cases to be used in the analysis. For additional information about our applied method we refer to an elaborate paper authored by our statistician Mamouris and coauthored by us. ${ }^{29}$

To clarify the concept of matching on comorbidities, consider a case with three chronic diseases the number of comorbidities equals three. When matching this case to a control, we allow for an absolute difference of one chronic disease, meaning that the controls could have two, three or four diseases. A total of 105 chronic diseases were taken into account for the operationalisation of comorbidity, of which 51 actually occurred in our study population. ${ }^{30}$

\section{Exposure and covariates}

To minimise the potential influence of a protopathic bias, prescriptions 1 year prior to the index date were not considered. The main exposure was defined as any oral antibiotic therapy during $1-10$ years prior to the index date, subdivided into the following eight classes (ATC code): tetracyclines (J01A), amphenicols (J01B), betalactam antibiotics, penicillines (J01C), other beta-lactam antibacterials (J01D), sulfonamides and trimethoprim (J01E), macrolides, lincosamides and streptogramins 


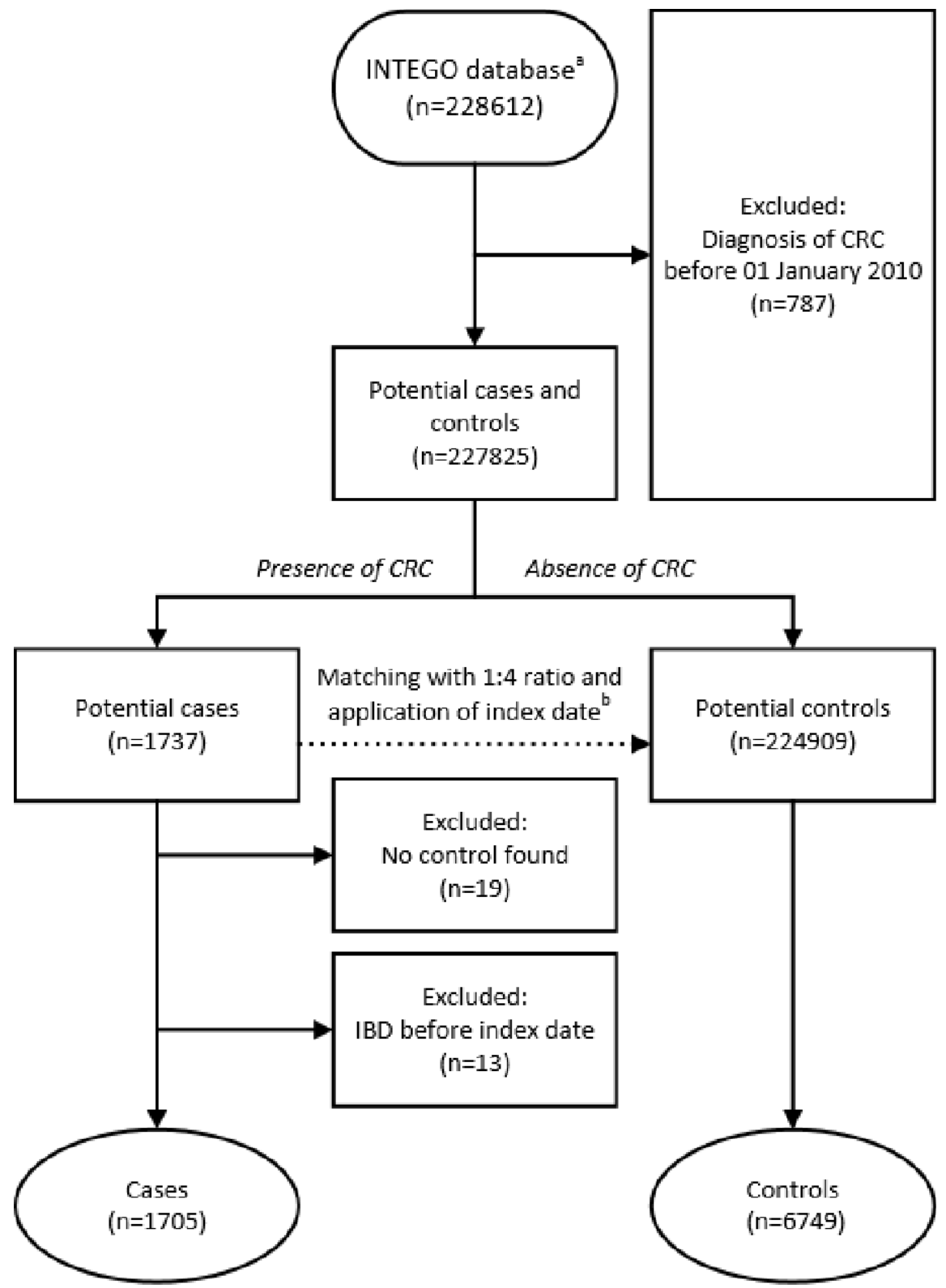

Figure 1 Patient selection flowchart. ${ }^{\text {T}}$ Total number of unique eligible subjects in the Intego database during 1 January 2010 until 31 December 2015. ${ }^{b}$ Controls with presence of IBD before the index date were excluded during matching. CRC, colorectal cancer; IBD, inflammatory bowel disease; Intego, Integrated Computerised Network.

(J01F), quinolone antibacterials (J01M) and other antibacterial drugs (J01X). Classes with aminoglycoside (J01G) and combinations of antibacterials (J01R) were not prescribed. These classes consist of chemical subgroups which categorise individual antibiotics on a molecular level. $^{31}$

The cumulative number of prescriptions per drug class prior to the index date were assessed. Multiple prescriptions prescribed during one consultation were added up only if they were of a different molecule. Subjects were categorised based on total number of prescriptions into the following categories: non-users, low (1st-33rd percentile), intermediate (34th-66th percentile), high (67th-90th percentile), very high (above 90th percentile) and all (1st percentile and above). Multiple categories might allow us to witness a dosage-response relationship. The presence of DM (ICPC-2 code T90) was taken into account as confounder. The main factor for which we lacked registration of sufficient quality and quantity was tobacco use. In addition certain genetic variants, such as Lynch syndrome, increase the chance of development of CRC. ${ }^{8}$ Due to missing details in the database about these often hereditary disorders we were not able to correct for these types.

\section{Statistical analysis}

The proportion of cases and controls using antibiotics, as well as the proportion of subjects using different classes of antibiotics were described. For continuous variables we used Student's t-test and Mann-Whitney U test. The 
Pearson $\chi^{2}$ test was used for categorical variables. The primary analysis was a conditional logistic regression analysis to estimate OR and 95\% CIs for the association between antibiotic prescriptions for oral use and the subsequent diagnosis of CRC. The multivariate analysis was conditioned on the presence of DM. Based on literature we decided to consider a minimal OR of 1.20 as a relevant risk factor. ${ }^{8}$ The predominance of the J01C class of $45 \%$ allowed us to estimate ORs of those who used one or more prescription of this class.

RStudio Team (2019) was used for statistical analysis. ${ }^{32}$

\section{Ethics statement}

The Intego procedures were approved by the ethical review board of the KULeuven Faculty of Medicine (no. ML 1723) and by the Belgian Privacy Commission (no. SCSZG/13/079).

\section{Patient and public involvement}

No patients were involved in the development of the research question, study design or interpretation of the data.

\section{RESULTS}

\section{Baseline characteristics}

The group of 1705 cases with a first diagnosis of CRC between 01 January 2010 and 31 December 2015 were matched to 6749 controls. Nineteen cases were excluded due to lack of any controls. As indicated in table 1, there were no statistically significant differences between the case and control groups in the terms used by matching. Due to the near perfect matching in terms of age and gender these factors were not corrected for during the analysis. The top three most prevalent comorbidities, which were similar in controls and cases, by most frequent

\begin{tabular}{|c|c|c|c|}
\hline & Cases & Controls & P value* \\
\hline $\begin{array}{l}\text { Absolute number of } \\
\text { subjects }\end{array}$ & 1705 & 6749 & \\
\hline $\begin{array}{l}\text { Mean age at index } \\
\text { date }( \pm \text { SD) }\end{array}$ & $58.85(13.48)$ & $58.53(14.11)$ & 0.40 \\
\hline $\begin{array}{l}\text { Male gender } \\
\text { (percentage) }\end{array}$ & 791 (46.39) & $3143(46.57)$ & 0.92 \\
\hline $\begin{array}{l}\text { Prevalence of } \\
\text { diabetes mellitus } \\
\text { (percentage) }\end{array}$ & 386 (22.64) & 877 (12.99) & $<0.01$ \\
\hline $\begin{array}{l}\text { Non-exposed } \\
\text { individuals } \\
\text { (percentage) }\end{array}$ & 675 (39.59) & 2915 (43.19) & $<0.01$ \\
\hline $\begin{array}{l}\text { Average number of } \\
\text { comorbidities per } \\
\text { subject }\end{array}$ & 2.35 & 2.29 & 0.35 \\
\hline
\end{tabular}

*Pearson $\chi^{2}$ test was used for categorical variables and Student's t-test for continuous variables. occurrence were hypertension, hypercholesterolaemia and asthma.

\section{Exposure}

In total 5217 antibiotic prescriptions were prescribed for the cases versus 18263 for the controls, resulting in a total average of respectively 3.06 and 2.71 per patient during the observation period of on average 9 years. This correlates to on average one prescription for every 3-year period for cases and on average one prescription every 3 -year and 4 months period for controls. The categorisation of cases and controls resulted in a combined group of non-users of 3590 individuals (42\% of total), 2110 in the low category (25\%), $1056(12 \%)$ in the intermediate category, $1188(14 \%)$ in the high exposure category and lastly $510(6 \%)$ in the very high group. The percentage non-users in the case group were a bit lower compared the control group ( $40 \%$ vs $43 \%, \mathrm{p}<0.01)$. The highest individual number of prescriptions was 122 prescriptions in total, which appeared a credible number after further investigation.

Figure 2 presents the relative share of the six most prescribed classes of antibiotics per year during the entire observational period. The major share consisted of beta-lactam antibacterials ( $45 \%$ on average), which in turn consisted of $89 \%$ of amoxicillin. Noteworthy is the gradual decline of other beta-lactam antibacterials, which consisted for over $90 \%$ of cefuroxime. Lastly there was an apparent increase of the class named other antibacterials, which mainly consisted of nitrofurantoin derivatives $(68 \%)$. Figure 3 presents the timewise use of prescriptions relative to the index date per case or control. As shown, there is a gradual increase in both the case and the control group. After the first year of measurement it shows a non-crossing pattern, with the cases consistently having a higher number of prescriptions.

\section{Association between antibiotics and risk of CRC}

Table 2 shows the main outcome of our study in terms of crude and adjusted ORs. The OR of developing CRC for antibiotic users with one or more prescriptions compared with non-users was 1.25 (95\% CI 1.10 to 1.44$)$. Due to the lack of a clear increase of ORs, the CIs of all categories were overlapping with their lesser-exposed category, an evident dose-response relationship could not be shown. The additional comparison of individuals who used one or more prescription of the penicillin and other betalactam antibacterials (J01C class) to non-users resulted in a crude OR of 1.13 (95\% CI 1.00 to 1.28). Due to this number being low and nearly insignificant we deemed an isolated effect of this subclass unlikely. Analysis of other classes did not result in significant ORs.

\section{DISCUSSION}

\section{Statement of the principal findings}

Antibiotic prescriptions compared with none were associated with a $25 \%$ higher chance of developing CRC over 


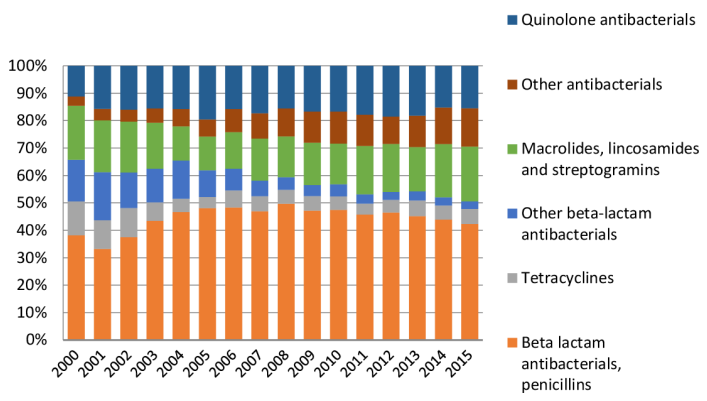

Figure 2 Relative antibiotic use per class per year (excluding two classes with $<2 \%$ of total prescriptions).

a 9-year period, a number that remained significant after correction for DM. All subdivided categories, except for low exposure (one to two prescriptions), showed a significantly increased OR. A dose-response relationship was not shown.

\section{Strengths and weaknesses}

The benefit of the Intego database was twofold. First the size of more than a quarter of a million of unique individuals during the selection period has allowed us to investigate a relatively rare disease. Second it has shown its representativeness for the Flemish-Belgian population in terms of the geological spread, age and sex. ${ }^{28}$ Exact and recent European statistics comparing outpatient and hospital prescriptions were not found. In the USA the GP, with $24 \%$ of all antibiotic prescriptions in 2011, accounted for the greatest share of all specialties. ${ }^{33}$ Considering the likelihood of the GP being the most frequent prescriber the impact of long-term side effects would be the greatest. Two unique features of our study were the use of an optimal matching procedure ${ }^{29}$ which optimised the use of controls and matching based on general disease burden using the comorbidity index.

The major limitation of this study is the absence of known risk factors, especially smoking. Furthermore two studies were found that indicated a lower threshold for doctors to prescribe antibiotics of certain classes for tobacco users. ${ }^{3435}$ Considering the increased relative risk of 1.20 (95\% CI 1.10 to 1.30) of developing CRC when comparing smokers to non-smokers found by Kelvin $e t a \varphi^{9}$ it is doubtful, but not excludable, that our found OR can fully be explained by smoking. One further limitation of

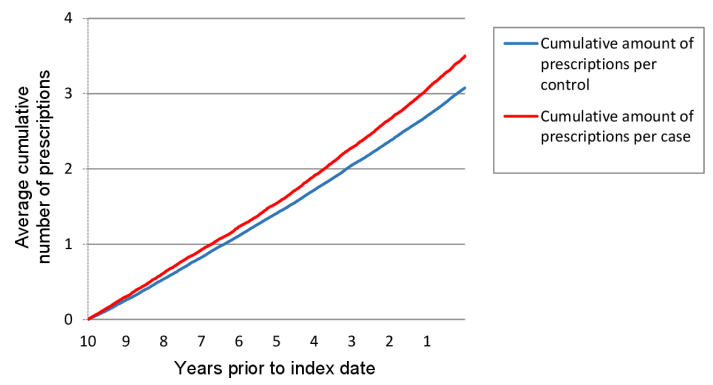

Figure 3 Average cumulative prescriptions per case or control prior to index date. *Observation period ranged from 1 to 10 years prior to the index date.
Table 2 Antibiotic prescriptions and OR of developing colorectal cancer

\begin{tabular}{lll}
\hline $\begin{array}{l}\text { Amount of } \\
\text { prescriptions } \\
\text { compared with } \\
\text { none* }\end{array}$ & OR (95\% Cl lower limit-upper limit) \\
\hline$>0$ & Crude & Adjustedt \\
\hline $1-2$ & $1.26(1.10$ to 1.45$)$ & $1.25(1.10$ to 1.44$)$ \\
\hline $3-4$ & $1.11(0.95$ to 1.31$)$ & $1.12(0.95$ to 1.32$)$ \\
$5-122$ & $1.37(1.07$ to 1.75$)$ & $1.36(1.06$ to 1.74$)$ \\
$5-10$ & $1.43(1.12$ to 1.82$)$ & $1.40(1.10$ to 1.79$)$ \\
$11-122$ & $1.31(1.01$ to 1.71$)$ & $1.30(1.00$ to 1.70$)$ \\
\hline
\end{tabular}

${ }^{*}$ Category (number of prescriptions): All $(>0)$, low (1-2), intermediate (3-4), high (5-10), very high (11-122). †Adjusted for presence of type 2 diabetes mellitus.

the used database is that Belgian patients are free to visit different GPs of their choice, possibly resulting in incomplete patient data regarding prescription of antibiotics and registration of relevant diagnoses. Lastly it should be stated that while a case-control study is often suited to test a certain hypothesis about the link between a risk factor and an outcome, its retrospective nature limits the power of investigating causality.

\section{Comparison to previous studies}

Comparing our results to similar studies, differences can be found in terms of investigated population, registration of exposure, diagnosis and method of correction for comorbidities. Our OR of any versus none users of 1.25 (95\% CI 1.10 to 1.44 ) is very similar to the pooled results from the meta-analysis of Simin $e t a l^{27}$ who found an OR of 1.17 (95\% CI 1.05 to 1.30 ). Ten studies lay the foundation for this meta-analysis, of which three were conducted in the USA ${ }^{15-17}$, five in Europe (UK N=3 ${ }^{19} 2122$, the Netherlands $\mathrm{N}=1^{14}$, Finland $\mathrm{N}=1^{20}$ ), one in New Zealand ${ }^{23}$ and one in Taiwan. ${ }^{18}$ In 2009 Belgium ranked sixth out of 33 countries for the amount of antibiotic prescriptions and was higher than the three aforementioned European countries, suggesting relatively high exposure in our study. ${ }^{13}$ Potential explanations for a positive correlation might be a causative relationship or potentially persistent lacking correction for a common confounder. A clear dose-response relationship was generally not considered shown, and lacking in our study. However, considering the described permanent effect of antibiotics on the microbiome ${ }^{6}$ it might behave more like a threshold type of model instead.

For detailed results comparable individual case-control studies are more suitable for comparison. Our OR when comparing our group with high exposure, meaning five or more prescriptions, to non-users was 1.40 (95\% CI 1.10 to 1.79). Dik et al established an OR of 1.23 (95\% CI 1.08 to 1.40) when comparing eight or more prescriptions versus none during 1-6 years prior to CRC diagnosis. ${ }^{14}$ Kilkkinen et al found an increased risk of developing 
CRC with an OR of 1.15 (95\% CI 1.04 to 1.26 ) when comparing six or more prescriptions versus $0-1$ during a follow-up period of 3-9 years after use. ${ }^{20}$ Our number is slightly higher, yet in line with these studies. In accordance with these studies ${ }^{1420}$ we did not find a significant relationship in our lowest exposure category (one to two prescriptions) when comparing to non-users. One study which corrected for smoking behaviour was by Armstrong et al. They found an increased OR of 1.90 (95\% CI 1.61 to 2.19) for the overall amount of prescriptions during the entire follow-up duration, which had a median of 6 years and a maximum of 15 years. ${ }^{21}$

\section{Recommendations for further research and clinical practice}

One possible explanation for our results might be the influence of antibiotics on the human gut, which contains a diverse microbial community and has a crucial role in the defence against pathogenic bacteria. ${ }^{4}$ Human studies with convincing evidence of a direct relationship between antibiotic use and CRC however are lacking. ${ }^{76}$ In order to study such a relationship one needs further investigation of the drug-microbiome relationship and its long-term effects. Considering the possibility of a permanent altered colonic state after antibiotic exposure, it is questionable whether this effect could result in a classical dose-response relationship or would behave as a threshold type model. The amount of exposure while we measured it seems relatively high in comparison to other countries. ${ }^{13}$ We consider our study, which was performed in a unique region by a unique matching procedure, has added weight to the hypothesis of an existent correlation between the exposure to oral antibiotics and CRC development. Its results highlight the value of additional research to improve the understanding of the interaction between antibiotics and CRC. Our results do support rational use, a trend which seems to become stronger due to the increased presence of antibiotic resistance. ${ }^{37}$ In our opinion additional clinical implications of our study are limited due to the inherent limitations of retrospective research.

\section{CONCLUSION}

In this case-control study, prescribed oral antibiotics predicted an increased risk of CRC without a clear dosageresponse relationship. The major limitation was lack of information on known risk factors of cancer, such as smoking. The retrospective observational nature warrants caution interpreting these results as proof of causality. Our inability of disproving the correlation between antibiotic exposure and development of CRC indicates the need of additional investigation. This study further supports the opinion of reserved and prudent usage of a potential lifesaving medicine.

\section{Author affiliations}

${ }^{1}$ Department of Public Health and Primary Care, Academic Centre for General Practice, KU Leuven Biomedical Sciences Group, Leuven, Flanders, Belgium
${ }^{2}$ Departement of Public Health and Primary Care, Katholieke Universiteit Leuven Groep Biomedische Wetenschappen, Leuven, Belgium

${ }^{3}$ Open Analytics NV, Antwerp, Belgium

${ }^{4}$ Department of Public Health and Primary Care, KU Leuven Biomedical Sciences Group, Leuven, Flanders, Belgium

${ }^{5}$ Institute of General Practice, Goethe University Frankfurt Faculty 16 Medicine, Frankfurt am Main, Hessen, Germany

Contributors JvdM: Conceptualisation, Methodology, Investigation, Writing original draft, Visualisation. PM: Formal analysis, Data curation, Software. VN: Formal analysis, Data curation, Software. BV: Conceptualisation, Methodology, Writing Review and Editing. MvdA: Conceptualisation, Methodology, Investigation, WritingReview and Editing, Supervision, Guarantor.

Funding Intego is funded on a regular basis by the Flemish Government (Ministry of Health and Welfare).

\section{Competing interests None declared.}

Patient and public involvement Patients and/or the public were not involved in the design, or conduct, or reporting, or dissemination plans of this research.

Patient consent for publication Not applicable.

Ethics approval Dossier number ethical accordance, KU Leuven, Belgium. The ethical commission of the UZ/ KU Leuven, Belgium, approved this study (MP004885).

Provenance and peer review Not commissioned; externally peer reviewed.

Data availability statement Data are available upon reasonable request. The Intego database is managed at the Department of Public Health and Primary Care of the University of Leuven under the supervision of Professor Dr Bert Vaes. The data set supporting the conclusions of this article is held at the University of Leuven, Belgium, and can be shared upon reasonable request.

Open access This is an open access article distributed in accordance with the Creative Commons Attribution Non Commercial (CC BY-NC 4.0) license, which permits others to distribute, remix, adapt, build upon this work non-commercially, and license their derivative works on different terms, provided the original work is properly cited, appropriate credit is given, any changes made indicated, and the use is non-commercial. See: http://creativecommons.org/licenses/by-nc/4.0/.

\section{ORCID iDs}

Johannes Van der Meer http://orcid.org/0000-0002-4109-5400

Bert Vaes http://orcid.org/0000-0001-5244-1930

Marjan van den Akker http://orcid.org/0000-0002-1022-8637

\section{REFERENCES}

1 Zaffiri L, Gardner J, Toledo-Pereyra LH. History of antibiotics. from Salvarsan to cephalosporins. J Invest Surg 2012;25:67-77.

2 Bartlett JG. Clinical practice. antibiotic-associated diarrhea. N Engl J Med 2002;346:334-9.

3 Song JH, Kim YS. Recurrent Clostridium difficile Infection: Risk Factors, Treatment, and Prevention. Gut Liver 2019;13:16-24.

4 Rinninella E, Raoul P, Cintoni M, et al. What is the healthy gut microbiota composition? a changing ecosystem across age, environment, diet, and diseases. Microorganisms 2019;7:14.

5 Borges-Canha M, Portela-Cidade JP, Dinis-Ribeiro M, et al. Role of colonic microbiota in colorectal carcinogenesis: a systematic review. Rev Esp Enferm Dig 2015;107:659-71.

6 Dethlefsen L, Relman DA. Incomplete recovery and individualized responses of the human distal gut microbiota to repeated antibiotic perturbation. Proc Natl Acad Sci U S A 2011;108 Suppl 1:4554-61.

7 Brenner $\mathrm{H}$, Chen $\mathrm{C}$. The colorectal cancer epidemic: challenges and opportunities for primary, secondary and tertiary prevention. $\mathrm{Br} \mathrm{J}$ Cancer 2018;119:785-92.

8 Brenner H, Kloor M, Pox CP. Colorectal cancer. Lancet 2014;383:1490-502.

9 Tsoi KKF, Pau CYY, Wu WKK, et al. Cigarette smoking and the risk of colorectal cancer: a meta-analysis of prospective cohort studies. Clin Gastroenterol Hepatol 2009;7:682-8.

10 Dong Y, Zhou J, Zhu Y, et al. Abdominal obesity and colorectal cancer risk: systematic review and meta-analysis of prospective studies. Biosci Rep 2017;37:1-12.

11 Ferlay J, Colombet M, Soerjomataram I, et al. Cancer incidence and mortality patterns in Europe: estimates for 40 countries and 25 major cancers in 2018. Eur J Cancer 2018;103:356-87. 
12 Cardoso R, Guo F, Heisser T, et al. Colorectal cancer incidence, mortality, and stage distribution in European countries in the colorectal cancer screening era: an international population-based study. Lancet Oncol 2021;22:1002-13.

13 Adriaenssens N, Coenen S, Versporten A. European surveillance of antimicrobial consumption (ESAC): outpatient antibiotic use in Europe (1997-2009). J Antimicrob Chemother 2011;66:3-12.

14 Dik VK, van Oijen MGH, Smeets HM, et al. Frequent use of antibiotics is associated with colorectal cancer risk: results of a nested Case-Control study. Dig Dis Sci 2016;61:255-64.

15 Falagas ME, Walker AM, Jick H, et al. Late incidence of cancer after metronidazole use: a matched metronidazole user/nonuser study. Clin Infect Dis 1998;26:384-8.

16 Friedman GD, Coates AO, Potter JD, et al. Drugs and colon cancer. Pharmacoepidemiol Drug Saf 1998;7:99-106.

17 Friedman GD, Jiang S-F, Udaltsova N, et al. Epidemiologic evaluation of pharmaceuticals with limited evidence of carcinogenicity. Int $J$ Cancer 2009;125:2173-8.

18 Wang J-L, Chang C-H, Lin J-W, et al. Infection, antibiotic therapy and risk of colorectal cancer: a nationwide nested case-control study in patients with type 2 diabetes mellitus. Int J Cancer 2014;135:956-67.

19 Boursi B, Haynes K, Mamtani R, et al. Impact of antibiotic exposure on the risk of colorectal cancer. Pharmacoepidemiol Drug Saf 2015;24:534-42.

20 Kilkkinen A, Rissanen $\mathrm{H}$, Klaukka T, et al. Antibiotic use predicts an increased risk of cancer. Int J Cancer 2008;123:2152-5.

21 Armstrong D, Dregan A, Ashworth M, et al. The association between colorectal cancer and prior antibiotic prescriptions: case control study. Br J Cancer 2020;122:912-7.

22 Zhang J, Haines C, Watson AJM, et al. Oral antibiotic use and risk of colorectal cancer in the United Kingdom, 1989-2012: a matched case-control study. Gut 2019;68:1971-8.

23 Didham RC, Reith DM, McConnell DW, et al. Antibiotic exposure and breast cancer in New Zealand. Breast Cancer Res Treat 2005;92:163-7.

24 Cao Y, Wu K, Mehta R, et al. Long-Term use of antibiotics and risk of colorectal adenoma. Gut 2018;67:672-678.

25 Sanyaolu LN, Oakley NJ, Nurmatov U, et al. Antibiotic exposure and the risk of colorectal adenoma and carcinoma: a systematic review and meta-analysis of observational studies. Colorectal Dis 2020;22:858-70.
26 Qu G, Sun C, Sharma M, et al. Is antibiotics use really associated with increased risk of colorectal cancer? an updated systematic review and meta-analysis of observational studies. Int $J$ Colorectal Dis 2020;35:1397-412

27 Simin J, Fornes R, Liu Q, et al. Antibiotic use and risk of colorectal cancer: a systematic review and dose-response meta-analysis. $\mathrm{Br} \mathrm{J}$ Cancer 2020;123:1825-32.

28 Truyers C, Goderis G, Dewitte H, et al. The Intego database: background, methods and basic results of a Flemish General practice-based continuous morbidity registration project. BMC Med Inform Decis Mak 2014;14:48.

29 Mamouris P, Nassiri V, Molenberghs G, et al. Fast and optimal algorithm for case-control matching using registry data: application on the antibiotics use of colorectal cancer patients. BMC Med Res Methodol 2021;21:1-9.

30 Shi X, Nikolic G, Van Pottelbergh G, et al. Development of multimorbidity over time: an analysis of Belgium primary care data using Markov chains and weighted association rule mining. $J$ Gerontol A Biol Sci Med Sci 2021;76:1234-41.

31 WHO Collaborating Centre for Drug Statistics Methodology. Guidelines for ATC classification and DDD assignment. 22 edn. WHO Collaborating Centre for Drug Statistics Methodology, 2019.

32 R Foundation for Statistical Computing, Vienna A. R Core Team (2019). R: A language and environment for statistical computing. [Internet], 2019. Available: https://www.r-project.org/

33 Suda KJ, Hicks LA, Roberts RM, et al. A national evaluation of antibiotic expenditures by healthcare setting in the United States, 2009. J Antimicrob Chemother 2013;68:715-8.

34 Steinberg MB, Akincigil A, Kim EJ, et al. Tobacco smoking as a risk factor for increased antibiotic prescription. Am J Prev Med 2016;50:692-8.

35 Blix HS, Hjellvik V, Litleskare I, et al. Cigarette smoking and risk of subsequent use of antibacterials: a follow-up of 365,117 men and women. J Antimicrob Chemother 2011;66:2159-67.

36 Dejea C, Wick E, Sears CL. Bacterial oncogenesis in the colon HHS public access. Futur Microbiol 2013;8:445-60.

37 ECDC. Surveillance of antimicrobial resistance in Europe (2017). [Internet]. Surveillance of antimicrobial resistance in Europe, 2018. Available: https://ecdc.europa.eu/en/publications-data/antimicrobialresistance-surveillance-europe-2016 